\title{
Relations of angiotensin-(1-7) with hemodynamic and cardiac structural and functional parameters in patients with hypertension and type 2 diabetes
}

\author{
Sergiy M. Koval', Kostyantin 0. Yushko', Iryna 0. Snihurska', Tetyana G. Starchenko', \\ Pankiv I. Volodymyr' ${ }^{2}$ Olga M. Lytvynova ${ }^{3}$, Olga V. Mysnychenko ${ }^{1}$ \\ 'Department of Arterial Hypertension and Prevention of Its Complications, L.T. Mala National Institute of Therapy of the NAMN Ukraine, \\ State Institution, Kharkiv, Ukraine \\ ${ }^{2}$ Department of Preventive Endocrinology, Ukrainian Scientific and Practical Center of Endocrine Surgery, Kyiv, Ukraine \\ ${ }^{3}$ Department of Laboratory Diagnostics, National University of Pharmacy, Kharkiv, Ukraine
}

\begin{abstract}
Background. The angiotensin-(1-7) is a new component of the renin-angiotensin system, the product of the degradation of angiotensin II and its functional antagonist, but its role in hypertension with type 2 diabetes (T2D) is not clear. The aim of the study was to investigate the levels of angiotensin-(1-7) in patients with hypertension and T2D and determine its relations to hemodynamic and cardiac structural and functional parameters.

Material and methods. We examined 70 patients with hypertension and T2D. Investigation protocol included physical examination, standard transthoracic echocardiography and determination of the angiotensin-(1-7) blood levels by ELISA. Control group consisted of 16 healthy volunteers.

Results. The angiotensin-(1-7) levels in observed patients were significantly lower than in volunteers [105.51 $(89.13 ; 121.17) \mathrm{ng} / \mathrm{L}$ vs. $132.75(125.06 ; 142.87) \mathrm{ng} / \mathrm{L}, \mathrm{p}<0.001]$. The levels of the angiotensin-(1-7) significantly negatively correlated with duration of hypertension $(\mathrm{r}=-0.29, \mathrm{p}<0.05)$, systolic blood pressure (BP) $(\mathrm{r}=-0.38, \mathrm{p}<0.05)$, diastolic BP $(\mathrm{r}=-0.36, \mathrm{p}<0.01)$, average BP $(\mathrm{r}=-0.32, \mathrm{p}<0.01)$, left ventricular (LV) internal dimension at end-diastole $(r=-0.37, p<0.01)$, LV mass $(r=-0.40, p<0.001)$, LV mass index $(r=-0.41$, $\mathrm{p}<0.001)$. In patients with LV hypertrophy, angiotensin-(1-7) levels were significantly lower than in patients without LV hypertrophy $[101.9(88.2 ; 117.7) \mathrm{ng} / \mathrm{L}$ vs. $129.3(117.5 ; 136.8) \mathrm{ng} / \mathrm{L}, \mathrm{p}<0.01]$ and in patients with diastolic LV dysfunction — lower than in the patients with normal diastolic function $[101.1(87.9 ; 116.6) \mathrm{ng} / \mathrm{L}$ vs. $121.1(105.5 ; 128.9) \mathrm{ng} / \mathrm{L}, \mathrm{p}<0.01]$.

Conclusions. The angiotensin-(1-7) can be considered as an important pathogenetic factor in the development of hypertension with T2D, a BP regulator and a cardioprotective agent that prevents the development of remodeling and diastolic dysfunction of the LV.
\end{abstract}

Key words: angiotensin-(1-7); hypertension; type 2 diabetes; left ventricular remodeling; diastolic dysfunction 


\section{Introduction}

Hypertension and type 2 diabetes (T2D) are the most world-spread non-infectious diseases. Even individually, each of them is associated with an increased risk of cardiovascular mortality. According to studies, more than $60 \%$ of patients with T2D have high blood pressure (BP). The prevalence of hypertension among patients with T2D reaches $49 \%$. It has been proved that T2D increases the cardiovascular risk 2-fold in men, 3-fold in women and the presence of hypertension further increases the risk 4 -fold in patients with diabetes [1]. Nowadays, the scientists pay special attention to studying the influence of hormones, adipokines, endogenous peptides, and inflammation factors in the pathogenesis of both hypertension and diabetes [2-4].

The leading pathogenetic role in the development of hypertension plays the renin-angiotensin system. In the classic sense, the renin-angiotensin system regulates the production of angiotensin II hormone from angiotensinogen. The renin and angiotensin-converting enzyme (ACE) type 1 is involved in this process, and the pathophysiological response to angiotensin II is mainly realized via angiotensin receptors type 1 [5]. At the same time, in recent years, the knowledge about the mechanisms for the involvement of renin-angiotensin system in the development of cardiovascular diseases has been expanded by discovering and studying the effects of angiotensin II metabolites, which include angioten$\sin -(1-7)[6]$.

Angiotensin-(1-7) is a heptapeptide which is derived from angiotensin II under the influence of ACE type 2. Angiotensin-(1-7) implements its physiological role through specific Mas receptors that are widely represented in human tissues [7] and acts as a functional antagonist of angiotensin II, has antihypertensive, antiremodeling, cardioprotective and antiatherosclerotic properties, affects the development of heart failure, ischemic heart disease, arrhythmias $[8,9]$. Presently, angiotensin-(1-7) and its synthetic analogs are considered as potential therapeutic agents in the treatment of a number of cardiovascular diseases [10].

Despite the great progress in understanding the role of angiotensin-(1-7) in cardiovascular disease, studies involving patients with both hypertension and T2D are few. Thus, the aim of the study was to investigate the levels of angiotensin-(1-7) in patients with hypertension and T2D and determine its relations to hemodynamic and cardiac structural and functional parameters.

\section{Material and methods}

\section{Study population}

The study involved 70 patients with grade $2-3$ hypertension combined with T2D in the Department of Hypertension of Government Institution "L.T. Malaya Therapy National Institute of the NAMS of Ukraine", Kharkiv. Definition and classification of hypertension, stratification of patients by total cardiovascular risk was performed according to "2013 ESH/ESC Guidelines" [11]. The T2D diagnosis was made according to "2013 ESC Guidelines on diabetes, pre-diabetes, and cardiovascular diseases developed in collaboration with the EASD" [12].

Inclusion criteria for the study were age of patients more than 18 years, presence of combination of hypertension with T2D, informed consent signed by the patient. Exclusion criteria were as follows: malignant hypertension, symptomatic hypertension, a history of myocardial infarction or stroke, secondary insulin-dependent diabetes, severe heart failure and coronary heart disease, congenital heart disease, acute or chronic systemic inflammatory conditions, severe pulmonary, liver and kidney diseases, known or treated malignancies, and pregnancy.

Age of the patients ranged from 40 to 70 years, median age was $57(52 ; 65)$ years. There were 34 man (48.6\%) and 36 women (51.4\%) among examined patients. Duration of hypertension in the patients was $15(10 ; 20)$ years, diabetes duration was $4(1 ; 6)$ years. Median level of systolic blood pressure (BP) was $170(170 ; 180) \mathrm{mmHg}$ and diastolic BP was $110(100 ; 110) \mathrm{mm} \mathrm{Hg}$. Nineteen patients $(27.1 \%)$ had grade 2 hypertension and ,51 patients (72.9\%) - grade 3 hypertension. Median level of glycated hemoglobin in patients with hypertension and T2D was $7.5 \%(7.1 \% ; 7.8 \%)$

The control group consisted of 16 healthy volunteers, who did not have history of any cardiovascular risk factors or illnesses.

\section{Study protocol}

All the participants were interviewed to obtain medical history. Physical examination was performed, including measurements of height, body weight, and waist circumference. The BP measuring was performed by auscultative method of Korotkov using a sphygmomanometer according to the standard method. The average BP was calculated as the sum of diastolic BP and one third of pulse BP. The pulse $\mathrm{BP}$ was defined as the difference between systolic and diastolic BP. 
Blood samples were drawn in the early morning from the antecubital vein of each subject after an overnight fasting period. Once drawn, the serum samples were centrifuged and immediately stored at $-20^{\circ} \mathrm{C}$ prior to the analysis. Levels of angiotensin-(1-7) were measured by enzyme-linked immunosorbent assay (ELISA), using ELISA kit of "YH Biosearch Laboratory", China and automated ELISA assay system "ImmunoChem — 2100" (HTI, USA) according to the manufacturer's instructions.

All subjects underwent standard trans-thoracic echocardiographic examination on the day of serum collection. The examinations were carried out using a "ULTIMA PA" echocardiography machine (RADMIR, Ukraine). All cardiac chamber measurements were made as suggested by the American Society of Echocardiography [13], including LV internal dimension at end-diastole (LVIDd), septal wall thickness at end-diastole (SWTd), posterior wall thickness at end-diastole (PWTd), left atrium (LA) anteroposterior linear dimension, diameter of the aortic root were measured using two-dimensional (2D) or M-mode images taken from parasternal long axis views of the heart. The LV ejection fraction was calculated using the LV volumes by the modified biplane Simpson's rule. LV mass (LVM) was calculated using the following formula:

$$
\begin{gathered}
L V M=0.80 \times\left(1 . 0 4 \left[(L V I D d+P W T d+S W T d)^{3}\right.\right. \\
\left.\left.-(L V I D d)^{3}\right]\right)+0.6 g
\end{gathered}
$$

Body surface area (BSA) was calculated by the Dubois formula:

$$
B S A=0.007184 \times \text { height }(\mathrm{cm})^{0.725} \times \text { weight }(\mathrm{kg})^{0.425}
$$

LVM index was calculated by dividing the LVM by the BSA. LVH was estimated using thresholds of $95 \mathrm{~g} / \mathrm{m}^{2}$ for women and $115 \mathrm{~g} / \mathrm{m}^{2}$ for men [11]. Calculation of relative wall thickness (RWT) by the formula, $(2 \times$ PWTd)/LVIDd, permits categorization of an increase in LV mass as either concentric $(\mathrm{RWT} \geq 0.42)$ or eccentric (RWT < 0.42) LVH and allows identification of concentric remodeling (normal LV mass with increased RWT). Normal LV geometry was determined as normal LV mass with normal RWT.

The diastolic function of LV was estimated from the data of the mitral inflow in pulsed-waved Doppler mode from the apical 4-chamber view. Primary measurements of mitral inflow include the peak early filling (E-wave) and late diastolic filling (A-wave) velocities, the E/A ratio, deceleration time of early filling velocity the isovolumetric relaxation time of the myocardium. An indication of early diastolic function impairment was considered with a decrease in E/A of less than 0.8 (type I diastolic dysfunction). When the normal values of diastolic function were detected, an additional analysis of the Valsalva maneuver was performed to determine the pseudonormal pattern (type II diastolic dysfunction).

\section{Statistical analysis}

The data were analyzed with IBM SPSS Statistics version 19 (SPSS for Windows, Version 19.0., SPSS Inc., Chicago, IL, USA). The distribution of variables normality was tested with the KolmogorovSmirnov test. The quantitative data were presented using median as a measure of position and quartile as a measure of dispersion. Continuous variables were defined as median (25 quartile; 75 quartile). The Mann-Whitney test was used to determine differences between groups. The Kruskal-Wallis dispersion analysis was applied for evaluation of influence of angiotensin-(1-7) levels on hemodynamics and cardiac indicators. The relationships between levels of angiotensin-(1-7) and other clinical variables were examined using Spearman's correlation. A value of $\mathrm{p}<0.05$ indicated a statistically significant result.

\section{Ethics statement}

The protocol of the study was carried out according to the principles of the Declaration of Helsinki and approved by local Ethics Committee (number: 03.04.2012/10058) [14]. Written informed consent was obtained from all the participants before enrolment.

\section{Results}

The blood levels of angiotensin-(1-7) in patients with hypertension and T2D were significantly lower than in healthy volunteers - $105.51(89.13 ; 121.17)$ ng/L vs. 132.75 (125.06; 142.87) ng/L, p < 0.001 (Fig. 1).

For Kruskal-Wallis dispersion analysis the levels of angiotensin-(1-7) were divided into three categories - more than $120.02 \mathrm{ng} / \mathrm{L}$ (norm), within the range of $100.79-120.01 \mathrm{ng} / \mathrm{L}$ (less than normal) and less than $100.79 \mathrm{ng} / \mathrm{L}$ (significantly less than normal). We evaluated the influence of angiotensin-(1-7) levels on hemodynamic and cardiac structural and functional parameters. The dispersion analysis results showed significant effect of blood angiotensin- $(1-7)$ levels on systolic BP $(\mathrm{p}<0.05)$, diastolic BP $(\mathrm{p}<0.01)$, average BP $(\mathrm{p}<0.01)$, 


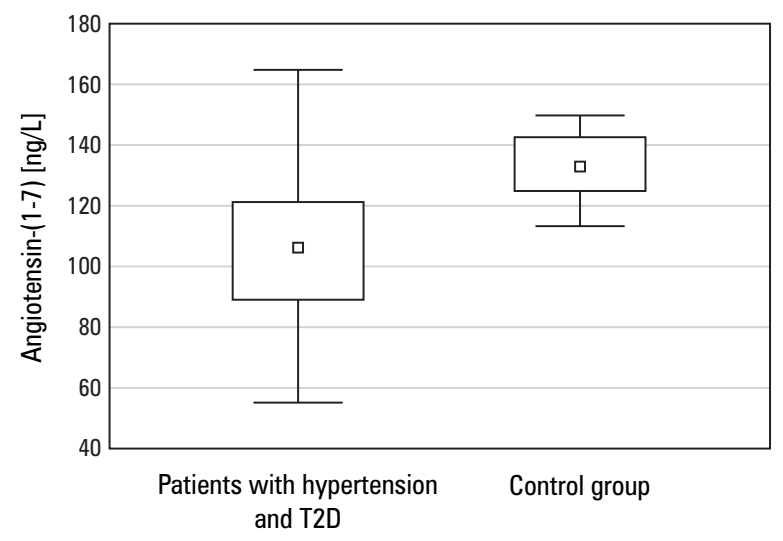

Figure 1. The angiotensin-(1-7) blood levels in patients with hypertension with type 2 diabetes (T2D) and in control group

Table I. Kruskal-Wallis dispersion analysis results

\begin{tabular}{|l|c|c|}
\hline Parameters & $\chi^{2}$ & $\mathbf{p}$ \\
\hline Systolic BP & 8.881 & $<0.05$ \\
\hline Diastolic BP & 9.663 & $<0.01$ \\
\hline Average BP & 11.723 & $<0.01$ \\
\hline Grade of hypertension & 8.974 & $<0.05$ \\
\hline LVIDd & 7.83 & $<0.05$ \\
\hline SWTd & 4.281 & $>0.05$ \\
\hline PWTd & 5.248 & $>0.05$ \\
\hline RWT & 8.45 & $<0.05$ \\
\hline LVM & 8.686 & $<0.05$ \\
\hline LVM index & 8.341 & $<0.05$ \\
\hline LA anteroposterior linear dimension & 5.604 & $>0.05$ \\
\hline Diastolic dysfunction & 11.829 & $<0.01$ \\
\hline Type of diastolic dysfunction & 17.031 & $<0.001$ \\
\hline
\end{tabular}

BP — blood pressure; LVIDd — left ventricular internal dimension at end-diastole; SWTd — septal wall thickness at end-diastole; PWTd — posterior wall thickness at end-diastole; RWT — relative wall thickness; LVM — left ventricle mass; LV — left ventricle; LA — left atrium

grade of hypertension $(\mathrm{p}<0.05)$, LVIDd $(\mathrm{p}<0.05)$, RWT ( $\mathrm{p}<0.05)$, LVM $(\mathrm{p}<0.05)$, LVM index $(\mathrm{p}<0.05)$, presence of diastolic dysfunction $(\mathrm{p}<0.01)$ and type of diastolic dysfunction $(\mathrm{p}<0.001)$ in patients with hypertension and T2D (Tab. I).

Correlation analysis revealed that in patients with hypertension and T2D the angiotensin-(1-7) levels significantly negatively correlate with duration of hypertension $(r=-0.29, p<0.05)$, hemodynamic parameters such as systolic BP $(r=-0.38$, $\mathrm{p}<0.05)$, diastolic BP $(\mathrm{r}=-0.36, \mathrm{p}<0.01)$, average BP $(\mathrm{r}=-0.32, \mathrm{p}<0.01)$ (Tab. II) and structural parameters of the LV such as LVIDd $(r=-0.37$, $p<0.01)$, $\operatorname{LVM}(\mathrm{r}=-0.40, \mathrm{p}<0.001)$, LVM index $(\mathrm{r}=-0.41$, $\mathrm{p}<0.001)$ (Tab. III).
Table II. The correlations of angiotensin-(1-7) with hemodynamic parameters

\begin{tabular}{|l|c|c|}
\hline Hemodynamic parameters & $\mathbf{r}$ & p \\
\hline Duration of hypertension & -0.29 & $<0.05$ \\
\hline Systolic BP & -0.38 & $<0.01$ \\
\hline Diastolic BP & -0.36 & $<0.01$ \\
\hline Average BP & -0.32 & $<0.01$ \\
\hline BP-blood pressure & &
\end{tabular}

Table III. The correlations of angiotensin-(1-7) with cardiac structural parameters

\begin{tabular}{|l|c|c|}
\hline Heart indicators & $\mathbf{r}$ & $\mathbf{p}$ \\
\hline LVIDd & -0.37 & $<0.01$ \\
\hline SWTd & -0.21 & $>0.05$ \\
\hline PWTd & -0.21 & $>0.05$ \\
\hline RWT & 0.10 & $>0.05$ \\
\hline LVM & -0.40 & $<0.001$ \\
\hline LVM index & -0.41 & $<0.001$ \\
\hline LV ejection fraction & 0.15 & $>0.05$ \\
\hline LA anteroposterior linear dimension & -0.19 & $>0.05$ \\
\hline
\end{tabular} $\begin{aligned} & \text { LVIDd - left ventricular internal dimension at end-diastole; SWTd - septal wall thickness at end-dia- } \\
& \text { stole; PWTd - posterior wall thickness at end-diastole; RWT - relative wall thickness; LVM - left } \\
& \text { ventricle mass; LV - left ventricle; LA - left tatrium }\end{aligned}$

We analyzed the levels of angiotensin-(1-7), depending on LV remodeling. In patients with LVH, angiotensin-(1-7) levels were significantly lower than in patients without LVH $[101.9(88.2 ; 117.7) \mathrm{ng} / \mathrm{L}$ vs. $129.3(117.5$; 136.8) ng/L, p < 0.01]. Depending on the type of LV remodeling in patients with concentric $\mathrm{LVH}$ and eccentric $\mathrm{LVH}$, the angiotensin-(1-7) levels were significantly lower compared with those with concentric remodeling of LV [107.5 (88.2; 119.6) $\mathrm{ng} / \mathrm{L}$ and $101.4(84.2 ; 111.8) \mathrm{ng} / \mathrm{L}$ vs. 129.3 (117.5; 136.8) ng/L, p $<0.01$ and $\mathrm{p}<0.01$ respectively], but probably did not differ from each other ( $p>0.05$ ) (Fig. 2).

Depending on the diastolic function of $\mathrm{LV}$ in patients with hypertension and T2D, the levels of angiotensin-(1-7) were significantly different in different subgroups. In the presence of diastolic LV dysfunction, the concentration of angiotensin-(1-7) was significantly lower than in the subgroup with normal diastolic function -101.1 (87.9; 116.6) $\mathrm{ng} / \mathrm{L} v s .121 .1(105.5 ; 128.9) \mathrm{ng} / \mathrm{L}(\mathrm{p}<0.01)$. At the same time, the levels of angiotensin-(1-7) were found to be the lowest in the subgroup with type II diastolic dysfunction and significantly different from its levels in the subgroup with normal diastolic function [97.2 (81.2; 105.4) ng/L vs. 121.1 (105.5; 128.9) ng/L, $\mathrm{p}<0.01]$ and in the subgroup with type I diastolic 


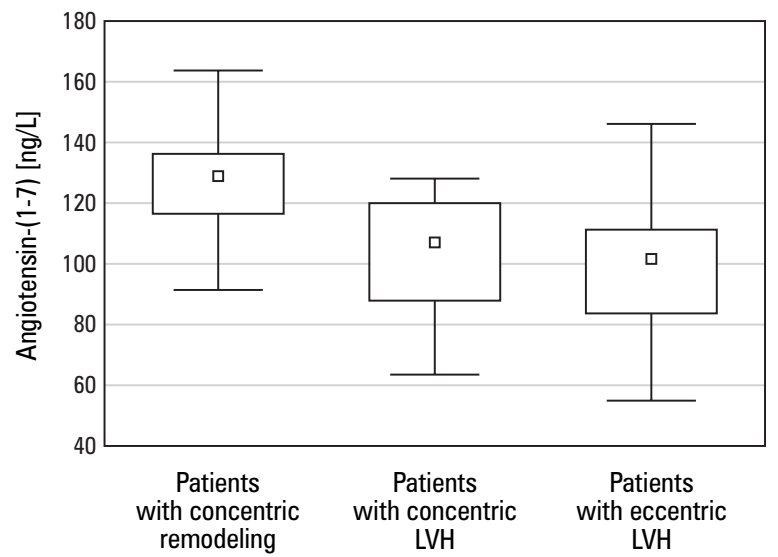

Figure 2. The angiotensin-(1-7) blood levels in patients with hypertension and type 2 diabetes (T2D) depending on the type of left ventricle (LV) remodeling; LVH — left ventricle hypertrophy

dysfunction [97.2 (81.2; 105.4) ng/L vs. 106.9 (88.2; 122.2) ng/L, $\mathrm{p}<0.05$ ]. In patients with type I diastolic dysfunction, the levels of angiotensin-(1-7) did not differ significantly from those in patients with normal diastolic function $(\mathrm{p}=0.07)$ (Fig. 3).

\section{Discussion}

The obtained results are of considerable scientific and practical interest. According to the literature data, the number of direct references to changes in levels of angiotensin-(1-7) in patients with hypertension is very limited. One study showed a fourfold decrease in levels of angiotensin-(1-7) in patients with hypertension with a certain ACE type 1 genotype [15]. According to other authors, the administration of angiotensin-(1-7) caused severe vasodilatation in patients with hypertension [16], which may indirectly indicate a deficiency of this peptide. Several experimental models show the important role of angiotensin-(1-7) in preventing systemic hypertension $[17,18]$ and vasodilation regulation $[19,20]$. In rats with hypertension, induction of diabetes caused a decrease in levels of angiotensin-(1-7), and increased levels prevented cardiovascular dysfunction, which develops in hypertension with diabetes [21]. In transgenic models of animals with overexpression of angiotensin-(1-7), induction of hypertension caused a decrease in levels of angiotensin-(1-7) in the peripheral blood flow with a local increase of this peptide in the heart [22]. In our study, there are clear associations of angiotensin-(1-7) deficiency with the development of hypertension in combination with T2D. Negative correlations of angiotensin-(1-7) levels with the

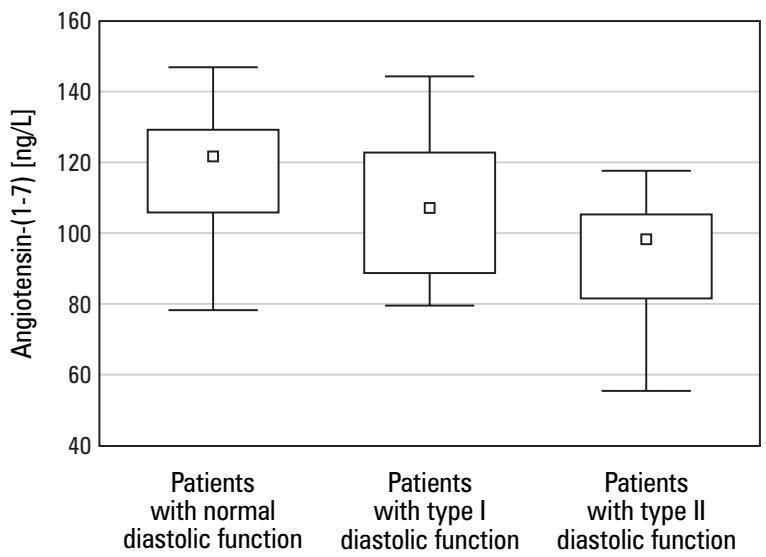

Figure 3. The angiotensin-(1-7) levels in patients with hypertension and type 2 diabetes (T2D) depending on diastolic function of left ventricle (LV)

main hemodynamic parameters indicate the regulatory role of angiotensin-(1-7) in BP.

We obtained interesting data about the relationship between the levels of angiotensin-(1-7) and pathological remodeling of LV in patients with hypertension and T2D. According to our results, the levels of angiotensin-(1-7) are decreased in patients with LVH and correlate negatively with LVIDd, LVM and LVM index, indicating cardiac antiremodeling properties of angiotensin-(1-7). This conclusion is confirmed by data from other studies. Patients with aortic stenosis and LVH have deficiency of angiotensin-(1-7) compared with healthy subjects [23]. In the experiment, it was shown that angiotensin-(1-7) decreases angiotensin II induced cardiac hypertrophy in rats $[24,25]$, including animals with insulin resistance [26], and prevents hyperglycemiainduced cardiomyocyte lesion [27].

The association of angiotensin-(1-7) with LV function has been shown in patients with T2D, in whom angiotensin-(1-7) levels were associated with both systolic and diastolic dysfunction [27]. Our study confirms this thesis as it was found that angiotensin-(1-7) levels depend on both the presence of diastolic dysfunction and its type. According to experimental data, hyperexpression of angiotensin-(1-7) prevents the occurrence of diastolic dysfunction and remodeling of LV in rats [21]. Cardioprotective mechanisms of angiotensin-(1-7) have not been fully understood, but scientists assume that they are associated with a decrease in oxidative stress and inflammation through the effect on the Mas receptors [28, 29].

Our study has several limitations. The present study has the relatively small number of patients. Angiotensin-(1-7) levels were measured only once during hospitalization. Its levels and relations with 
cardiac parameters in response to different variants of antihypertensive treatment were not evaluated. We could not assess cardiac remodeling and cardiovascular events depending on basal angiotensin-(1-7) concentrations in long-term period.

Despite the limitations of this study, it appears that angiotensin-(1-7) can be considered as an important pathogenetic factor in the development of hypertension with T2D, a BP regulator and a cardioprotective agent that prevents the development of remodeling and diastolic dysfunction of the LV.

\section{References}

1. Petrie JR, Guzik TJ, Touyz RM. Diabetes, Hypertension, and Cardiovascular Disease: Clinical Insights and Vascular Mechanisms. Can J Cardiol. 2018; 34(5): 575-584, doi: 10.1016/j.cjca.2017.12.005, indexed in Pubmed: 29459239.

2. Lau WB, Ohashi K, Wang Y, et al. Role of Adipokines in Cardiovascular Disease. Circ J. 2017; 81(7): 920-928, doi: 10.1253/circj. CJ-17-0458, indexed in Pubmed: 28603178.

3. Brzeska A, Sołtysiak M, Ziemak J, et al. Plasma adiponectin in hypertensive patients with and without metabolic syndrome. Arterial Hypertension. 2018; 22(1): 29-36, doi: 10.5603/ah.a2017.0025.

4. Koval S, Iushko K, Starchenko T. Relations of Apelin with Cardiac Remodeling in Patients with Hypertension and Type 2 Diabetes. Folia Med (Plovdiv). 2018; 60(1): 117-123, doi: 10.1515/folmed-2017-0066, indexed in Pubmed: 29668460.

5. Wu CH, Mohammadmoradi S, Chen JZ, et al. Renin-Angiotensin System and Cardiovascular Functions. Arterioscler Thromb Vasc Biol. 2018; 38(7): e108-e116, doi: 10.1161/ATVBAHA.118.311282, indexed in Pubmed: 29950386.

6. Patel VB, Zhong JC, Grant MB, et al. Role of the ACE2/Angiotensin 1-7 Axis of the Renin-Angiotensin System in Heart Failure. Circ Res. 2016; 118(8): 1313-1326, doi: 10.1161/CIRCRESAHA.116.307708, indexed in Pubmed: 27081112.

7. Karnik SS, Singh KD, Tirupula K, et al. Significance of angiotensin 1-7 coupling with MAS1 receptor and other GPCRs to the renin-angiotensin system: IUPHAR Review 22. Br J Pharmacol. 2017; 174(9): 737-753, doi: 10.1111/bph.13742, indexed in Pubmed: 28194766.

8. Santos RA. Angiotensin-(1-7). Hypertension. 2014; 63(6): 11381147, doi: 10.1161/HYPERTENSIONAHA.113.01274, indexed in Pubmed: 24664288.

9. McKinney CA, Fattah C, Loughrey CM, et al. Angiotensin-(1-7) and angiotensin-(1-9): function in cardiac and vascular remodelling. Clin Sci (Lond). 2014; 126(12): 815-827, doi: 10.1042/CS20130436, indexed in Pubmed: 24593683.

10. Machado-Silva A, Passos-Silva D, Santos RA, et al. Therapeutic uses for Angiotensin-(1-7). Expert Opin Ther Pat. 2016; 26(6): 669-678, doi: 10.1080/13543776.2016.1179283, indexed in Pubmed: 27121991.

11. Mancia G, Fagard R, Narkiewicz K, et al. Task Force Members. 2013 ESH/ESC Guidelines for the management of arterial hypertension: the Task Force for the management of arterial hypertension of the European Society of Hypertension (ESH) and of the European Society of Cardiology (ESC). J Hypertens. 2013; 31(7): 1281-1357, doi: 10.1097/01.hjh.0000431740.32696.cc, indexed in Pubmed: 23817082.

12. Rydén L, Grant PJ, Anker SD, et al. Authors/Task Force Members, ESC Committee for Practice Guidelines (CPG), Document Reviewers. ESC Guidelines on diabetes, pre-diabetes, and cardiovascular diseases developed in collaboration with the EASD: the Task Force on diabetes, pre-diabetes, and cardiovascular diseases of the European Society of Cardiology (ESC) and developed in collaboration with the European Association for the Study of Diabetes (EASD). Eur
Heart J. 2013; 34(39): 3035-3087, doi: 10.1093/eurheartj/eht108, indexed in Pubmed: 23996285.

13. Lang RM, Bierig M, Devereux RB, et al. American Society of Echocardiography's Nomenclature and Standards Committee, Task Force on Chamber Quantification, American College of Cardiology Echocardiography Committee, American Heart Association, European Association of Echocardiography, European Society of Cardiology. Recommendations for chamber quantification. Eur J Echocardiogr. 2006; 7(2): 79-108, doi: 10.1016/j.euje.2005.12.014, indexed in Pubmed: 16458610 .

14. Rickham PP. Human experimentation. Code of Ethics of the World Medical Association. Declaration of Helsinki. Br Med J. 1964; 2(5402): 177, doi: $10.1136 / \mathrm{bmj} .2 .5402 .177$, indexed in Pubmed: 14150898

15. Jalil JE, Palomera C, Ocaranza MP, et al. Levels of plasma angiotensin-(1-7) in patients with hypertension who have the angiotensinI-converting enzyme deletion/deletion genotype. Am J Cardiol. 2003; 92(6): 749-751, doi: 10.1016/s0002-9149(03)00847-6, indexed in Pubmed: 12972127.

16. Sasaki S, Higashi $Y$, Nakagawa K, et al. Effects of angiotensin-(1-7) on forearm circulation in normotensive subjects and patients with essential hypertension. Hypertension. 2001; 38(1): 90-94, indexed in Pubmed: 11463766.

17. Shi Y, Lo CS, Padda R, et al. Angiotensin-(1-7) prevents systemic hypertension, attenuates oxidative stress and tubulointerstitial fibrosis, and normalizes renal angiotensin-converting enzyme 2 and Mas receptor expression in diabetic mice. Clin Sci (Lond). 2015; 128(10): 649-663, doi: 10.1042/CS20140329, indexed in Pubmed: 25495544.

18. Benter IF, Yousif MHM, Anim JT, et al. Angiotensin-(1-7) prevents development of severe hypertension and end-organ damage in spontaneously hypertensive rats treated with L-NAME. Am J Physiol Heart Circ Physiol. 2006; 290(2): H684-H691, doi: 10.1152/ ajpheart.00632.2005, indexed in Pubmed: 16403946.

19. Raffai G, Lombard JH. Angiotensin-(1-7) Selectively Induces Relaxation and Modulates Endothelium-Dependent Dilation in Mesenteric Arteries of Salt-Fed Rats. J Vasc Res. 2016; 53(1-2): 105-118, doi: 10.1159/000448714, indexed in Pubmed: 27676088.

20. Yousif MHM, Benter IF, Diz DI, et al. Angiotensin-(1-7)dependent vasorelaxation of the renal artery exhibits unique angiotensin and bradykinin receptor selectivity. Peptides. 2017; 90: 10-16, doi: 10.1016/j.peptides.2017.02.001, indexed in Pubmed: 28192151

21. Li He, Liu X, Ren Z, et al. Effects of Diabetic Hyperglycemia on Central Ang-(1-7)-Mas-R-nNOS Pathways in Spontaneously Hypertensive Rats. Cell Physiol Biochem. 2016; 40(5): 1186-1197, doi: 10.1159/000453172, indexed in Pubmed: 27960152.

22. Santiago NM, Guimarães PS, Sirvente RA, et al. Lifetime overproduction of circulating Angiotensin-(1-7) attenuates deoxycorticosterone acetate-salt hypertension-induced cardiac dysfunction and remodeling. Hypertension. 2010; 55(4): 889-896, doi: 10.1161/HYPERTENSIONAHA.110.149815, indexed in Pubmed: 20212262

23. López-de la Vega C, Rosas-Peralta M, Lomelí-Estrada C, et al. [Urinary levels of angiotensin-(1-7) and angiotensin II in patients with severe aortic stenosis]. Arch Cardiol Mex. 2011; 81(4): 292-297, indexed in Pubmed: 22188883.

24. Guo L, Yin A, Zhang Qi, et al. Angiotensin-(1-7) attenuates angiotensin II-induced cardiac hypertrophy via a Sirt3-dependent mechanism. Am J Physiol Heart Circ Physiol. 2017; 312(5): H980-H991, doi: 10.1152/ajpheart.00768.2016, indexed in Pubmed: 28411231

25. Wang $\mathrm{Lj}$, He Jg, Ma H, et al. Chronic administration of angiotensin-(1-7) attenuates pressure-overload left ventricular hypertrophy and fibrosis in rats. Di Yi Jun Yi Da Xue Xue Bao. 2005; 25(5): 481-487, indexed in Pubmed: 15897115.

26. Giani JF, Muñoz MC, Mayer MA, et al. Angiotensin-(1-7) improves cardiac remodeling and inhibits growth-promoting pathways in the heart of fructose-fed rats. Am J Physiol Heart Circ Physiol. 2010; 298(3): H1003-H1013, doi: 10.1152/ajpheart.00803.2009, indexed in Pubmed: 20061544. 
27. Lei $Y, X u Q$, Zeng Bo, et al. Angiotensin-(1-7) protects cardiomyocytes against high glucose-induced injuries through inhibiting reactive oxygen species-activated leptin-p38 mitogen-activated protein kinase/extracellular signal-regulated protein kinase $1 / 2$ pathways, but not the leptin-cJun N-terminal kinase pathway in vitro. J Diabetes Investig. 2017; 8(4): 434-445, doi: 10.1111/jdi.12603, indexed in Pubmed: 27896943.

28. Hao PP, Chen YG, Liu YP, et al. Association of plasma angiotensin-(1-7) level and left ventricular function in pa- tients with type 2 diabetes mellitus. PLoS One. 2013; 8(5): e62788, doi: 10.1371/journal.pone.0062788, indexed in Pubmed: 23690953.

29. Lin $\mathrm{Li}, \mathrm{Liu} \mathrm{X}, \mathrm{Xu}$ J, et al. Mas receptor mediates cardioprotection of angiotensin-(1-7) against Angiotensin II-induced cardiomyocyte autophagy and cardiac remodelling through inhibition of oxidative stress. J Cell Mol Med. 2016; 20(1): 48-57, doi: 10.1111/ jcmm.12687, indexed in Pubmed: 26515045. 